\title{
iPSC technology-Powerful hand for disease modeling and therapeutic screen
}

\author{
Changsung Kim ${ }^{*}$ \\ Department of Bioscience and Biotechnology, Sejong University, Seoul 143-747, Korea
}

\begin{abstract}
Cardiovascular and neurodegenerative diseases are major health threats in many developed countries. Recently, target tissues derived from human embryonic stem (hES) cells and induced pluripotent stem cells (iPSCs), such as cardiomyocytes (CMs) or neurons, have been actively mobilized for drug screening. Knowledge of drug toxicity and efficacy obtained using stem cell-derived tissues could parallel that obtained from human trials. Furthermore, iPSC disease models could be advantageous in the development of personalized medicine in various parts of disease sectors. To obtain the maximum benefit from iPSCs in disease modeling, researchers are now focusing on aging, maturation, and metabolism to recapitulate the pathological features seen in patients. Compared to pediatric disease modeling, adult-onset disease modeling with iPSCs requires proper maturation for full manifestation of pathological features. Herein, the success of iPSC technology, focusing on patient-specific drug treatment, maturation-based disease modeling, and alternative approaches to compensate for the current limitations of patient iPSC modeling, will be further discussed. [BMB Reports 2015; 48(5): 256-265]
\end{abstract}

\section{INTRODUCTION}

Conventional disease modeling studies, using animal models and immortalized cell systems, have been consistently used to study disease pathology and therapeutic development (1). Artificially manipulated cells have been used to screen therapies for monogenic diseases, such as cancer, neurodegenerative diseases, and congenital heart disease (2). Although monogenic diseases have been actively studied to identify cures, multiple mutations, as seen in compound-mutation diseases, have not been analyzed for drug screening. It has been challenging to

${ }^{*}$ Corresponding author. Tel: +82-2-3408-4485; Fax: +82-2-34084334; E-mail: changkim@sejong.ac.kr

http://dx.doi.org/10.5483/BMBRep.2015.48.5.100

Received 9 May 2014, Revised 15 June 2014,

Accepted 4 August 2014

Keywords: Disease modeling, Drug screen, IPSC, Patient specific therapy, Stem cell design disease models that involve multiple genes and mimic most human diseases $(1,3)$. Interestingly, $95 \%$ of new drugs screened using artificially manipulated cells were withdrawn due to off-target effects (4).

To alleviate the problems associated with off-target effects, studies using human tissues have been suggested. However, studies using human samples pose some major challenges. First, it is difficult to obtain fresh human disease samples. Second, it is not possible to critically observe the development of pathological features, since most of the human samples are obtained from the end stages of the disease or postmortem. Hence, human iPSC disease modeling has emerged as an alternative modeling method for obtaining both important human pathology data and unlimited resources. Since 2008, there has been rapid development of iPSC-mediated disease modeling and novel therapy options (5) (Table 1). Human iPSC disease modeling has key advantages compared to its predecessors. For example, it provides a complicated genetic signature of human tissue and an unlimited resource for identifying the mechanism underlying pathology development and for developing novel cures.

In this review, I have discussed the success obtained with congenital and sporadic disease modeling using iPSCs for representative diseases. Furthermore, the importance of tissue-specific maturation in disease modeling has been discussed. Last, the importance of hES- and iPSC-based drug screening in cardiology, collectively human cell-based screening, has been addressed with respect to reducing unwanted toxicity issues and obtaining an amenable outcome for new therapies.

\section{OVERVIEW OF iPSC}

There has been massive interest in the application of iPSCs since studies by Dr. Yamanaka first reported mouse and human iPSC generation $(6,7)$. The LIF-dependent mouse iPSC and bFGF-based human iPSC cultures differed from each other, but their basis for transformation was identical $(6,7)$. For example, Oct3/4, Sox2, Klf4, and c-Myc (Yamanaka factors) were used as transforming factors in both cases. Initially, a retrovirus was used to generate iPSCs. Later, inducible lentiviruses, mRNAs, DNA episomes, peptides, and non-integrating viruses, such as the Sendai virus, were developed to generate iPSCs (8). 
Table 1. Successful human iPSC mediated therapy cases

\begin{tabular}{|c|c|c|c|c|c|}
\hline Disease & Mutations & $\begin{array}{l}\text { Disease pathology } \\
\text { found }\end{array}$ & $\begin{array}{l}\text { Disease mechanism } \\
\text { found }\end{array}$ & $\begin{array}{l}\text { Patient specific } \\
\text { therapy suggested }\end{array}$ & Reference \\
\hline Leopard syndrome & PTPN11 & YES & NA & NA & (22) \\
\hline LQT1 & KCNQ1 (R190Q) & YES & NA & NA & (16) \\
\hline LQT2 & KCNH2 (A614V) & YES & NA & NA & (17) \\
\hline LQT8 & CACNA1C & YES & NA & NA & (18) \\
\hline LQT3- & SCN5A (F1473C), KCNH2 (K897T) & YES & YES & YES* & (20) \\
\hline ARVD/C & $P K P 2$ & YES & YES & NA & (55) \\
\hline Brugada & PKP2 & YES & YES & NA & (78) \\
\hline Schizophrenia & Not identified & YES & YES & YES & (32) \\
\hline$A D$ & Sporadic \& familial (APP) & YES & YES & YES & (28) \\
\hline AD & PS1 (A246E) \& PS2 (N141I) & YES & YES & YES & (29) \\
\hline AD & Sporadic \& APP (duplication) & YES & YES & YES & (31) \\
\hline ALS & SOD1 (+/A4V), SOD1 (+/D90A) & YES & YES & YES & $(48,49)$ \\
\hline SMA & SMN1 & YES & YES & YES & $(44,46)$ \\
\hline $\mathrm{HD}$ & HTT & YES & YES & YES** & $(52,53)$ \\
\hline PD & PINK, Parkin, LRRK2 & YES & YES & YES & $(54,56)$ \\
\hline RTT & MECP2 & & YES & YES & (37) \\
\hline PWS & Chromosome 15q11-q13 deletion & YES & NA & NA & (43) \\
\hline Hepatic disorder & AAT & YES & YES & YES** & (62) \\
\hline
\end{tabular}

*Living patient therapy has been made, **Novel drug screen has been made, NA: Not applicable, PS1: Presenilin-1, PS2: Prsenlilin-2, APP: Amyloid Precursor Protein, PKP2: Plakophilin-2.

Both ethical and translational obstacles in hES cell-based human therapies were overcome by Yamanaka's iPSC technology. The potential to generate many types of vital tissues and cells, such as hematopoietic stem cells, heart muscles, and motor neurons, brought worldwide attention to the potential of this patient-specific cell-based therapy (9).

Researchers envision the use of iPSCs in two main ways: as a source for cell-based therapy without immune rejection (9) and as a tool for disease modeling to identify patient-specific cures (5). In this review, successful cases of iPSC disease modeling have been presented, and suggestions for drug screening on the basis of stem cell-based tests have been discussed.

\section{DIFFERENTIATION OF CARDIAC MUSCLE FROM iPSCs}

Initial stem cell-based CM differentiation was influenced by early frog embryonic development. During gastrulation, the embryo differentiates into three layers, which further develop as the endoderm, ectoderm and mesoderm (10). Early endoderm and mesoderm induction is influenced by Nodal, which is a family member of Wnt and TGF- $\beta$. Endodermal influence on cardiac differentiation was confirmed by artificially adding endoderm into the early-stage embryo to induce ectopic heart tissue formation (11).

By mimicking early embryonic development, a three-dimensional embryonic body (3D EB) with serum-mediated spontaneous cardiac differentiation was obtained; this has been well documented (12). Moreover, adding endodermal lineage cells (END-2 co-culture) with early differentiating hES cells increased the efficiency of heart muscle differentiation up to $\sim 50 \%$ compared to the $\sim 20 \%$ efficiency obtained with the spontaneous 3D EB method (13). In 2008, Keller's group reported the involvement of BMP4 and DKK/Wnt signaling (14) in the induction of cardiac muscle with defined media. In addition, Kamp's group demonstrated robust CHIR99201/IWP4mediated Wnt/ $\beta$-catenin regulation, with up to $98 \%$ cardiac muscle generation from hES cells and iPSCs (15).

\section{SUCCESSFUL PEDIATRIC CARDIAC DISEASE MODELING}

\section{Long QT (LQT)}

LQT syndrome is one of the cardiac diseases that were initially studied extensively using iPSCs. A series of LQT1, LQT2, and LQT8 iPSC models has been reported since late 2010 and 2011 (16-18). LQT has 12 different subsets of categories depending on the mutations and phenotypes (19). In all three early models of LQT iPSCs, clinically available regimens, as well as novel proof-of-concept options, were tested in vitro. However, regimen usage and therapeutic efficacy trials in living patients were not reported. Recently, iPSC disease modeling was successfully used in relation to a therapeutic regimen in the clinic for an LQT3 patient with compound mutations (20).

Conventional treatment of LQT3 involves blocking an abnormally activated $\mathrm{Na}^{+}$current $(19,21)$. When the $\mathrm{Na}^{+}$channel blockers mexiletine and flecanide were used to block the $\mathrm{Na}^{+}$current, delayed repolarization and a reduced prolonged QT interval was seen in the LQT patient (21). A study reported that the patient did not respond to any available clinical regi- 
mens before the study had begun (20); however, by increasing the beating frequency, it was observed that inactivation of the $\mathrm{Na}^{+}$current increased and the proarrhythmic activity decreased. Furthermore, this study confirmed that an iPSC-mediated therapeutic regimen was also successful with a living patient (20).

\section{ADULT ONSET CARDIAC DISEASE MODELING}

\section{LEOPARD syndrome}

The first success in iPSC disease modeling in adult-heart disease was Leopard syndrome (22). As shown in adult heart hypertrophy, iPSC-derived CM of Leopard syndrome became bigger compared to the control CMs. Within 30 days of cell culture in regular cardiogenic media, the patient's CM became notably bigger and more hypertrophic. This was a great success in adult-onset disease, which allowed for the study of a devastating human adult-heart disease in a much shorter time frame. In addition, from the pathology development standpoint of view, it brought the researchers' interest in adult onset disease modeling. It was a dramatic hypertrophic feature for the Leopard syndrome iPSC CM within 30 days in culture, since patients typically develop the disease pathology in their late 40 s.

\section{Hypertrophy}

Lan et al. reported reprogramming modeling with hypertrophic cardiomyopathy patient-derived iPSC (23). In this study, they could not find any pathological behavior while growing regular cardiogenic culture with 3D EB forms. When singlet culture of CM was performed in indefinite stiffness plate, it revealed abnormal $\mathrm{Ca}^{2+}$ transients and arrhythmic activity, revealing delayed after depolarization (DAD) (23). Their finding brought important insight of arrhythmia disease community to accelerate adult onset disease in a dish. The residing mechanism of singlet-cultures in a dish induced hypertrophic response would be resourceful.

\section{MORE DISEASE MODELING USING iPSCs}

\section{EARLY SUCCESS OF DISEASE MODELING USING iPSC: NEURONAL DISEASE}

It is worthwhile to mention the early success history of disease modeling, such as neuronal disease, since the first suggestion of iPSC disease modeling was based on neurodegenerative disease (5). Neuronal differentiation is one of the initially differentiated target-tissue from various sources of stem cells, such as hES, hiPSC, and somatic stem cells (24). Three major protocols have been mobilized to generate neurons, such as 3D EB based spontaneous method without serum, co-culture based method, and direct-induction of neurons with various chemicals and growth factors method (24-27). Due to future therapeutic usage, various attempts to induce neurons form hES and hiPSC have been made. Upon neuronal-rosette formation after short EB-based serum free induction, CNS neurons mostly resided within rosette, and migrating PNS neurons (descendant of neural crest cells) could be separated based on their developmental property. Moreover, by adding inhibitors of TGF and BMP signaling, researchers have obtained enhanced efficiency of neuronal induction (27). Proper subtype neuronal-lineage differentiation from iPSCs has allowed researchers to decipher various pathological features to find potential cures.

\section{Alzheimer's Disease (AD)}

AD iPSC modeling is one of the most actively studied neurological disorders using iPSCs (28-30). AD is an adult-onset disease, and the underlying pathology development is not well understood. Less than $10 \%$ of the patients have familial mutations, such as Presenilin-1 (PS1), Presenilin-2 (PS2), and Amyloid Precursor Protein (APP) (Table 1).

Recently, researchers found that iPSC-derived neurons from different patients showed different pathophysiology of $A D$, especially in the accumulation of $A \beta$ oligomers $(28,31)$. A $\beta$-oligomer-accumulation is one of the hallmarks of $A D$ development. This indicates the unsolved mechanism of $A \beta$ oligomers behavior in $A D$ patients. Israel et al. reported that only one sporadic AD patient iPSC neuron presented accumulation of $A \beta$ oligomers (31). In both the Kondo et al. and Israel et al. reports, A $\beta$-oligomer accumulating neurons from individual iPSC showed increased ER-stress and cell damages due to increased reactive-oxygen-species (ROS). Although $A \beta$-oligomer-accumulation is not correlated with the patients' genetic background, treatment for those affected neurons can be predicted through iPSC modeling. Furthermore, those iPSC-derived neuron data were well correlated with the patient's regimen-data, such as DHA treatment to ameliorate ROS-mediated cell damage. AD patient iPSC-derived neuron can be a good therapeutic testing ground to find the patient's optimal pharmacological regimen.

\section{Schizophrenia (SCZD)}

Schizophrenia (SCZD) study by Brennand et al. is one of the hallmark studies for genetically unidentified patient cases using iPSC disease modeling. In their study, they combined the data from a child-onset SCZD patient (6 year old) and 3 adult-onset SCZD patients (32). They found that genetically unknown SCZD patient samples had similar pathological signature. Both types of patients showed reduced neuritis number, postsynaptic-protein-95 (PSD95) expressions, and glutamate receptor expressions. They suggested that SCZD might share different pathological mechanisms. One candidate is the altered developmental signaling, such as Notch and Wnt signaling. It could be interpreted to have a common prevention method of SCZD among genetically-un-known SCZD patients.

More iPSC-based neuronal disorders studies have been reported in genetically identified cases. Amyotrophic lateral sclerosis (ALS), spinal muscular atrophy (SMA), Parkinson's disease (PD), Huntington disease (HD), Rett Syndrome (RTT), and Prader-Willi syndrome (PWS) are on going subjects of therapeutic screen (33-47). 


\section{Amyotrophic lateral sclerosis (ALS)}

Multiple groups reported ALS iPSC disease modeling $(48,49)$. Two recent reports used Superoxide-Dismutase-1 (SOD1) mutated patient fibroblast, which covers $20 \%$ of familial ALS cases, to generate iPSC. Kiskinis et al. performed high resolution RNA seq from SOD1 $1^{+/ A 4 V}$ dominant mutation iPSC and ZFNmediated isogenic control iPSC-derived MNs to narrow down the pathogenic mechanism (48). The authors found that motor neurons (MNs) from SOD1 $1^{+/ A 4 V}$ dominant-mutation iPSC showed that the expressions of mitochondrial-related and ER-stress-mediated genes were reduced. Their genetic analysis was confirmed by mitochondrial behavior analysis and ER stress response. Furthermore, the pathogenic feature of $S O D 1^{+/ A 4 V}$ ALS patient iPSC MNs was found to be conserved in different genetic background, such as the GGGGCC repeat expansions in C9orf72 locus patients' iPSC MNs.

Chen et al. found the autonomous behavior of mutated SOD1 in MNs, but not in non-MNs (49). Only in MNs, mutated SOD1 $1^{+/ D 90 A}$ was found to bind to the $3^{\prime}$ UTR region of neurofilament (NF)-L mRNA and interfere with the association of NF with NF-H and NF-M, resulting in NF aggregations in MNs. By restoring NF-L expression, mutated SOD $1^{+/ D 90 A}$ iPSC MNs mitigated neurite degeneration. Further elucidating the ALS pathogenic mechanism by those efforts could potentially bring a novel cure for these patients.

\section{Spinal muscular atrophy (SMA)}

SMA is also a MN disease, which showed elevated MN death. Survival-motor-neuron-1 (SMN1) gene mutation causes SMA $(44,46)$. Sareen et al. reported that SMA patient iPSC MNs showed elevated cell death, which was accompanied with prolonged activation of caspase 3. By blocking apoptosis, they were able to improve the neurological feature of SMA MNs, indicating common treatment options for SMA.

Corti et al. introduced a different approach to SMA treatment. SMA pathology is due to homozygous mutation in SMN1; however, most patients have normal SMN2. One of the therapeutic concepts to SMA was increasing SMN1-like proteins in patient-motor neurons. If SMA patient cells were treated with VPA or tobramycin, then the intrinsic SMN2 stop codon can be bypassed to make SMN1-like products (50). In addition, treatment with a GSK3-kinase inhibitor in ES-derived MNs showed significant increase of SMN, indicating plausible outcome for future human SMA treatment (51). But, Cori et al. provided gene-edited SMN2 to mimic SMN1 in patient iPSC MNs. Their proof-of-concept trials with the gene-edited MNs transplant rescued the SMA mutant mice (46).

\section{Huntington disease (HD)}

$\mathrm{HD}$ is an autosomal dominant neurodegenerative disease caused by a cytosine-adenine-guanine (CAG) repeats in the first exon of Huntington (HTT) gene. Several HD iPSCs disease modeling were reported with deepened-understanding of HD pathology development, such as mitochondria fragmentation and BDNF dependency for survival $(34,36,47)$. Recently, mitochondria-fragmentations-blocker treatment and BNDF over-activation through RE-1-silencing-transcription factor (REST) regulation showed alleviated disease pathology (52, 53). Those efforts could potentially bring therapeutic benefit to many patients suffering from $\mathrm{HD}$, which has no cure so far.

\section{Rett Syndrome (RTT)}

Rett syndrome (RTT) patients suffer devastating neurodevelopmental delay, mostly in females (41). RTT is caused by a mutation in methyl-CpG-binding-protein-2 (MECP2), which is highly expressed in mature neurons and glia (37). iPSC disease modeling on RTT has been reported. Furthermore, recent progress in MECP2 and IGF1 correlation could potentially bring about therapy for RTT patients $(37,40)$. For instance, Williams et al. rescued RTT patient iPSC-derived neurons with either IGF1 or GPE (a peptide with first 3 amino acids of IGF1) treatment, indicating future therapeutic options for RTT (37).

\section{Prader-Willi syndrome (PWS)}

PWS is a disease of genetic-imprinting, especially in paternal chromosome 15q11-q13 region (39). Also, large deletion of the paternal chromosome 15q11-q13 during meiosis induces PWS (43). PWS patients suffer from neurological disorder and early-child-hood obesity. Recent generation of PWS iPSC may facilitate therapeutic suggestion for the patients, since no mice model reproduces the whole feature of PWS (43).

\section{EVOLVING CONCEPT OF IPSC DISEASE MODELING}

\section{Maturation-based disease modeling}

Recently, two reports have been published regarding maturation-based adult on-set disease modeling: one was on Parkinson's disease (PD) and the other on the cardiac disease arrhythmogenic right ventricular dysplasia/cardiomyopathy (ARVD/C) $(54,55)$.

\section{Progerin-based maturation in PD modeling}

PD is one of the highly studied iPSC disease models and one of the most common neurodegenerative diseases in ageing populations $(47,54,56)$. In PD, increased cell death is seen in dopaminergic neurons (DNs) with complicated genetic stress, such as mutations in $\alpha$-synuclein, leucine-rich repeat kinase 2 (LRRK2), PTEN-induced putative kinase 1 (PINK), and parkin, as well as with environmental stress.

However, late-onset disease models using iPSCs often show incomplete penetrated pathology from patient iPSC-derived target tissues. Aged cells showed loss of the heterochromatin markers trimethylated $\mathrm{H} 3 \mathrm{~K} 9$ ( $\mathrm{H} 3 \mathrm{~K} 9 \mathrm{me} 3)$ and heterochromatinprotein-1 $\gamma(\mathrm{HP} 1 \gamma)$, but they became rejuvenated during iPSC transformation. It is often difficult to find pathological differences in embryonic stage patient iPSC DNs, considering their naïve neuronal stage. Miller et al. combined progerin, the protein associated with Hutchinson-Gilford progeria syndrome (HGPS), with PINK1-Q456X- and Parkin-V324A-mutated iPSCs 
to manifest robust PD pathology in vitro (54). In progeria, the lamin A in the nuclear membrane has a defect and accumulates in aged or HGPS patients. By ectopically inducing a progerin peptide in patient iPSC derived DNs, they detected robust pathological phenomena, such as loss of dendrites and increased cell death.

\section{Metabolic maturation-based disease modeling}

Recently, we analyzed patient-derived iPSC disease models in ARVD/C with metabolic maturation and noted adult-onset pathophysiological features (55). ARVD/C is an inherited cardiomyopathy that can cause sudden-cardiac-death in young adults, and a majority of these patients have the desmosome mutation (55). iPSCs from the fibroblasts of an ARVD/C patient with a homozygous c. $2484 \mathrm{C}>\mathrm{T}$ mutation in plakophilin-2 (PKP2) were established. Surprisingly, ARVD/C patient-derived iPSC CMs initially did not show significant abnormalities, such as cell death or lipid accumulation (55).

As cardiac muscle matures, energy metabolism switches from glucose-based non-aerobic ATP generation to aerobic and fatty acid-based $\beta$-oxidation (57). During postnatal development, the main hormones (e.g., insulin, adrenalin, and thyroid hormone) and cholesterol provide essential cues for the proper maturation and development of various organs. Cardiac energy metabolism shifts from glycolysis-based to lipid-based oxidation with the induction of three factors, that is, insulin, adrenalin, and cholesterol (55). Ectopic activation of PPAR-gamma (PPAR $\gamma$ ) was observed in the heart of an ARVD/C patient (58). By inducing PPAR $\gamma$, in addition to three factor-mediated metabolic maturation, we manifested pathological phenotypes of ARVD/C hearts within $\sim 60$ days in culture (55). Simple PPAR $\gamma$ over-activation without presetting metabolically matured hES/patient iPSC-derived CMs did not induce pathological development, indicating that maturation was a prerequisite for the development of adult pathology.

\section{Tissue culture stiffness on proper maturation}

Tissue culture stiffness is a crucial factor that affects cell biology and physiology during stem cell differentiation in many tissues, such as pancreatic cells, osteoblast differentiation, and CMs (59). Studies with neonatal rat CMs showed that indefinite stiffness disrupted the regular heart-beat, even after a short period in culture (60). Human tissues, such as heart tissues, provide stiffness (approximately 10-17 $\mathrm{kPa}$ ) to individual cardiac muscles, which supports normal beating and growth of the muscle $(60,61)$. To verify abnormal behaviors in LEOPARD syndrome, further correlation studies using hypertrophic iPSC-derived CMs, and tissue culture stiffness, which are important for abnormal growth and activity of tissues, may provide a valuable adult-onset iPSC disease modeling platform $(22,23)$.

\section{ADVANTAGES OF USING STEM CELL-DERIVED TISSUES FOR FUTURE DRUG SCREENING}

Screening drugs using iPSCs has major advantages over immortalized cell- or small animal-based screening methods, since it provides patient-specific efficacy. Efficacy and toxicity can be studied using human-derived iPSCs to minimize unwanted side effects. Furthermore, knowledge gained from iPSC disease models can provide new therapeutic options for complicated human diseases involving multiple genes, which cannot be applied with conventional disease modeling. There have been a handful of successful drug testing and efficacy trials using iPSCs in recent reports.

\section{Novel drug candidate screen for hepatic disorders}

Choi et al. recently used iPSCs from patients with alpha antitrypsin (AAT) deficiency to drive hepatocytes and to screen 3131 compounds and reported novel drug candidates for hepatic disorders (62). The most common AAT deficiency was due to a point mutation in the AAT gene, which induced spontaneous polymerization, ER protein overload in hepatocytes, and liver disorders. They found five candidates that decreased the accumulation of the mutant form of AAT in cells; all these candidates have been approved by the FDA for different diseases. Interestingly, one of their candidates, carbamazepine (CBZ), showed great efficacy in reducing hepatic fibrosis in the AAT mouse model, indicating the durability and efficacy of iPSC-based drug screening (63).

\section{Loxapine for SCZD}

The cause of SCZD is not known and treatment results for patients are variable. One interesting result from the Brennand et al. study provided important insights into the molecular basis of antipsychotic drugs, such as clozapine, loxapine, olanzapine, risperidone, and thioridazine, with SCZD iPSC neurons (32). Interestingly, one of the candidate drugs, loxapine, increased neuritis and the connectivity between neurons, explaining a possible therapeutic mechanism for SCZD. Although their studies were limited to a small number of patient iPSC lines, this human disease iPSC model allowed for the discovery of optimized treatment options for a group of patients with genetically unidentified disease.

\section{Kenpaullone for ALS}

Using stem cell-derived MNs, researchers have tried to identify substances related to MN-specific survival (64). Recently, Yang and colleagues reported a novel therapeutic regimen for ALS, using SOD1 mouse ES MNs and human ALS patient iPSC-derived MNs (65). Kenpaullone was one of the candidates that increased the survival of SOD1 mouse-derived MNs, and its efficacy was tested in human SOD1 iPSC-derived MNs. Although their initial screen was not based on iPSC MNs from ALS patients, it clearly proved to be a successful beginning for drug screening in ALS. 


\section{Human iPSC-based therapeutic safety consideration} Cardiac toxicity is an imminent factor for newly developed drugs. Recently, many newly developed and even market-available drugs have been retracted due to cardiac sudden death with QT interval increase and tachycardia-based arrhythmia attack, mostly resembling cardiac LQT syndrome. For example in 1996, an anti histamine drug, Seldane, was retracted from the market after it was attributed to 98 death cases in the United States. In addition, in early 2000 more than 8 non-cardiac post-market drugs were retracted due to severe cardio toxicity (66).

Conventional cardiac toxicity test has been performed with immortalized $\mathrm{CHO}$ cell based $\mathrm{IC}_{50}$ human ether-a-go-go-related gene ( $\mathrm{hERG}=I_{k r}$, rapid component inward rectifying $\mathrm{K}^{+}$ current) activity test for possible QT interval elongation (66, 67). Anti-HERG activity from unsafe drugs is the major cause of cardiac arrest and arrhythmia (68). QT interval elongation is the key cardiac toxicity criteria in newly invented drugs for human trials (66), and it is an important indicator for imminent threat to patients. QT interval is the time between Q and T waves during cardiac beating, and it is called electrocardiogram (ECG) recording. QT can be generally explained as the ventricular action-potential-duration (APD), such as beginning of pump in and end of pump out in the ventricle.

Many researchers suspect that the cause of cardio toxicity problem in newly developed non-cardiac drugs is due to immortalized cell based drug toxicity screen, since those cells accumulate mutations and are immune to newly developed drug-induced toxicity (69). Pharmaceutical companies often waste valuable resources due to adverse outcome of toxicity problem. In fact, the cost of QT interval toxicity check has been attributed to about $22 \%$ of the cost in phase 1 human trials in United States (67). These cases show the importance of human-oriented toxicity test. To avoid non-physiological drug candidates screen from immortalized cells with single protein over-expression setting, researchers tried human tissues, such as human heart muscle tissues, to test novel drugs.

However, it is very difficult to obtain fresh samples from patients. More often, those samples are not useful due to sample preparation errors. Liang and colleagues' recent report (69) revealed two major findings in stem cell-based toxicity studies and iPSC-based novel therapy screen. They found that the sensitivity of $I_{k r} \mathrm{~K}^{+}$current inhibitions among various pro-arrhythmogenic drugs was more reliable in hES/iPSC-derived $\mathrm{CM}$, rather than in hERG-expressing $\mathrm{CHO}$ or 293 cell lines. They showed that hERG blockage from a market-available safe drug, verapamil, was observed in a 293 cell-based toxicity study, whereas cardiac toxic Alfuzocin did not show severe toxicity in the same setting (69). In other words, safe drugs could be dismissed and dangerous drugs replaced, if researchers did not use hES/iPSC-derived CM for toxicity test. In human heart muscle, hundreds of ion channels are expressed and orchestrated. Only human ES/iPSC-derived CM faithfully mimics the milieu of human ion channels in the human heart (70).

It could be a perfect drug screening system if it can solve the drug efficacy and safety issues with the same cell-based platform. Patient iPSC-derived target tissue-based drug screening answers both needs and gives us better opportunity to find therapy (Fig. 1).
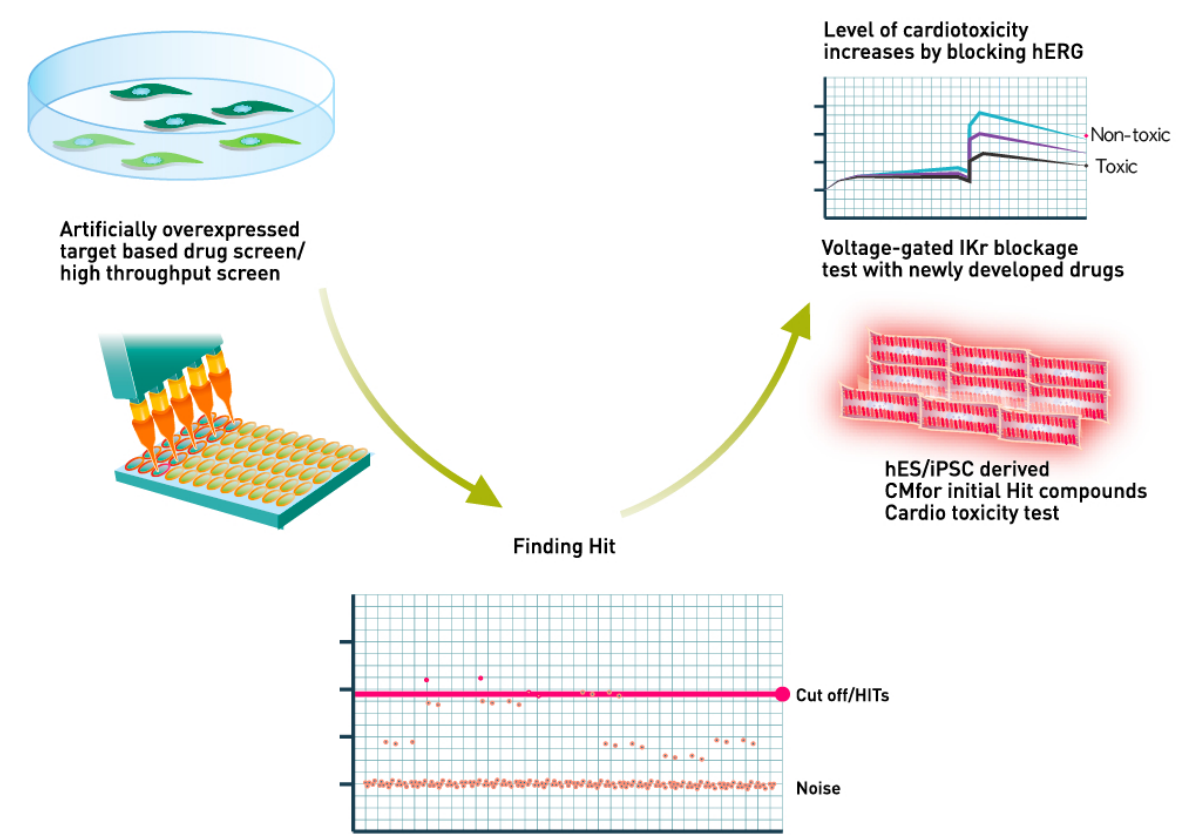

Fig. 1. Schematic view of novel hES/ iPSC-derived CM-based human cardiac toxicity test. $I_{k r}$ (rapid component of inward rectifying $\mathrm{K}^{+}$current, $\mathrm{hERG}$ ) is the major category to correlate possible human cardio toxicity. Decrease of hERG by drugs indicating the increase of QT interval by drugs, which induce severe cardiotoxic event. 


\section{LIMITATION OF iPSC-MEDIATED DISEASE MODELING AND RECENT PROGRESS}

One of major criticisms of iPSC disease modeling is the limited numbers of comparison groups (71). Two alternative methods can solve this potential limitation of iPSC disease modeling; One is the gene editing-mediated disease modeling by inducing mutation in normal iPSC, and the other is the direct conversion from somatic source of patient samples to target tissues, such as neuron or CM.

\section{Gene editing-based disease modeling}

For human patient-derived iPSC generation, human internal review board (IRB) approval often delays or even completely blocks the study progress. Furthermore, genetically affected human tissues are very difficult to obtain for statistically significant studies. If researchers can make mutated stem cells in their own laboratory, using available normal hESs/hiPSCs, then robust studies can be conducted. There has been a movement to generate allele specific patient iPSC lines without the original samples from the patients (72). Gene editing technology provides various options to fix or mutate certain gene or genes without tracing exogenous genome. Currently, RNA-guided-engineered-nuclease (RGENs, derived from clustered-regularly-interspaced-short-palindromic-repeat (CRISPR)-Cas), zincfinger-nuclease (ZFN), and transcription-activator-like-effectornuclease (TALEN)-based disease modeling are widely utilized $(73,74)$.

However, gene-edited hES/iPSC may not reproduce full-blown human pathology as patient sample-derived iPSC. Disease pathology often pre-requires the patients' life styles and complicated genetic modifiers, which only patient-derived iPSC could provide. Gene editing-mediated disease modeling from various academic institutes should be studied together with disease causing modifiers in order to make fully recapitulating human disease modeling.

\section{Direct conversions-based disease modeling}

Direct conversion is also much faster than conventional iPSCs, which require labor- and time-intensive generation/ characterization/ tests. We can avoid these numerical issues with direct tissue conversion methods. Direct conversions from patient fi-

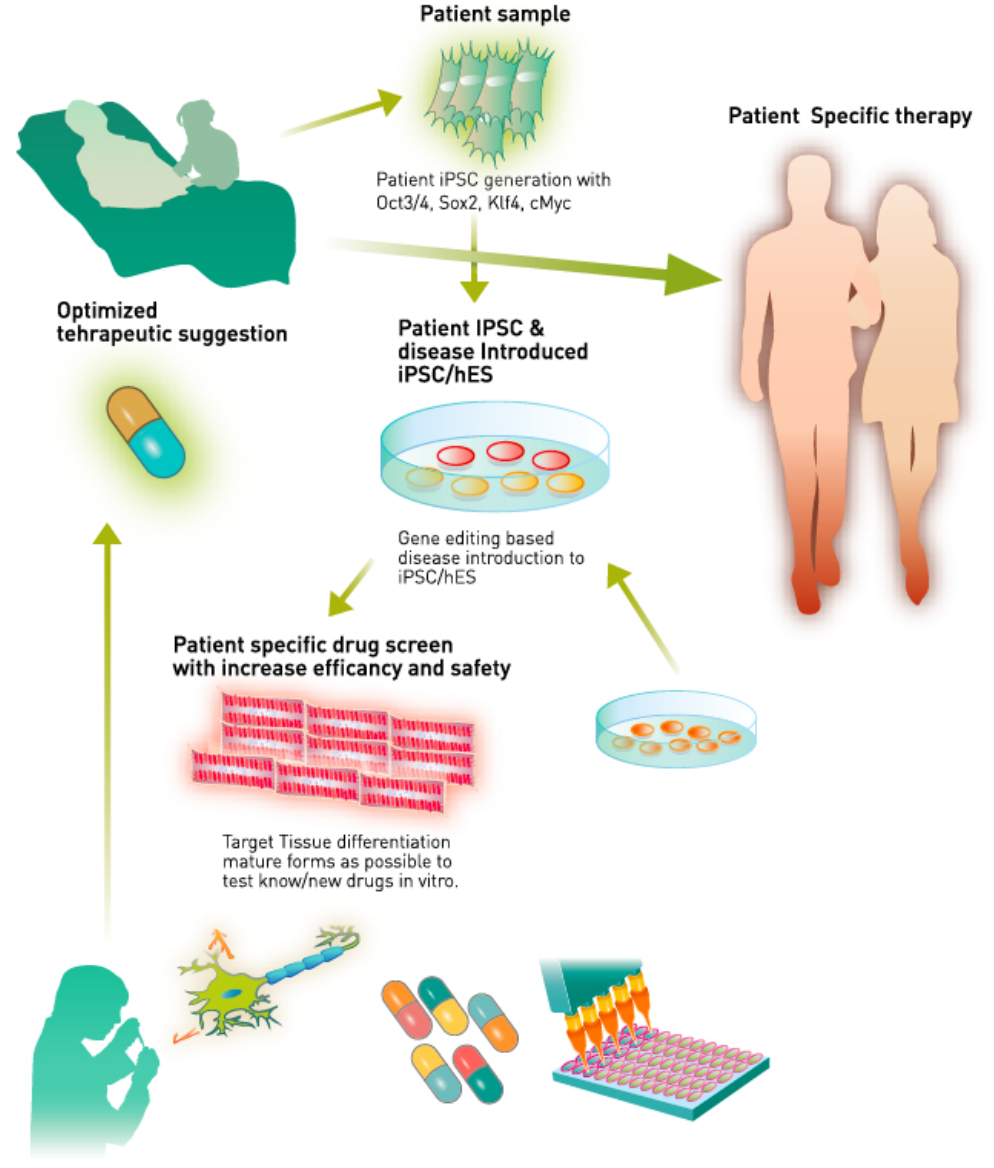

Fig. 2. Human patients and patient gene edited iPSC/hES-mediated novel therapy development. Disease containing stem cells were differentiated into target tissue such as neurons and CM. Adult-stage-maturated target tissue will be used to screen novel therapeutic candidates or market-available drugs to find right dosage for individual patients. Novel drugs' cardiotoxicity test will be performed while optimizing the efficacy of target hit. Optimized therapy will be pursued to live human patients. 
broblast to neurons have been reported recently (75). Currently, more researchers would like to use human-induced neurons (hiNs)-mediated neurological disease modeling, instead of generating hiPSCs, since there are advantages to using hiNs over hiPSC (75). First, we can increase the sample numbers from a few iPSC lines to a few thousand of hiNs. Second we can focus disease studies only rather than stem cell study. Cardiac disease also became the follower to use direct conversion as tools to study disease $(76,77)$. However, there may be unnecessary stress during direct conversion from human patient samples, and it needs to be redirected to the disease modeling. Direct conversion can be the next solution for disease modeling, as long as the conversion process does not put unnecessary stress to the cells or cause unexpected molecular diversion, which could interfere with tissue identity and pathological development.

\section{CONCLUSION}

iPSCs are being used to develop patient-specific therapies and their use has been successful for many diseases. These patient-specific medical regimens have been considered quite successful. Compared to the success of the pediatric disease model, adult-onset disease modeling faces hurdles and challenges such as disease recapitulation and relevant therapy test issues (71). However, the recent success in adult-onset disease in ARVD/C and PD cases can be models for the ongoing iPSC-derived disease modeling community $(54,55,78)$.

The key steps to identifying novel therapies include both generating target tissues from patient iPSCs and obtaining mature adult-like tissues. In addition to maturation setting, we can test novel pathophysiological approaches for generating patient-specific medicine. Studies involving international collaboration and increased numbers of patient samples and iPSC quality controls would greatly facilitate the development of novel therapies (Fig. 2). Furthermore, a combination of direct conversion-based disease models, such as hiN from patient tissue or gene editing-based mutation introduction, can strengthen the potential of stem cell-based disease modeling. We expect an unprecedented amount of important evidence to accumulate in the search for the cure for diseases.

\section{ACKNOWLEDGEMENTS}

This research was supported by Basic Science Research Program through the National Research Foundation of Korea (NRF) funded by the Ministry of Education (2014R1A1A2054906) and the Original Technology Research Program funded by the Ministry of Science and Technology (2014M4A9E5073759). I thank Drs. Han Choe and Hee Chul Lee for providing critical reading.

\section{REFERENCES}

1. Rajamohan D, Matsa E, Kalra S et al (2013) Current status of drug screening and disease modelling in human pluripotent stem cells. Bioessays 35, 281-298

2. Rubin LL and Haston KM (2011) Stem cell biology and drug discovery. BMC Biol 9, 42

3. Seok J, Warren HS, Cuenca AG et al (2013) Genomic responses in mouse models poorly mimic human inflammatory diseases. Proc Natl Acad Sci U S A 110, 3507-3512

4. Munos B (2009) Lessons from 60 years of pharmaceutical innovation. Nat Rev Drug Discov 8, 959-968

5. Park IH, Arora N, Huo H et al (2008) Disease-specific induced pluripotent stem cells. Cell 134, 877-886

6. Takahashi K, Tanabe K, Ohnuki M et al (2007) Induction of pluripotent stem cells from adult human fibroblasts by defined factors. Cell 131, 861-872

7. Takahashi K and Yamanaka S (2006) Induction of pluripotent stem cells from mouse embryonic and adult fibroblast cultures by defined factors. Cell 126, 663-676

8. Ban $\mathrm{H}$, Nishishita N, Fusaki $\mathrm{N}$ et al (2011) Efficient generation of transgene-free human induced pluripotent stem cells (iPSCs) by temperature-sensitive Sendai virus vectors. Proc Natl Acad Sci U S A 108, 14234-14239

9. Kim C (2014) Disease modeling and cell based therapy with iPSC: future therapeutic option with fast and safe application. Blood Res 49, 7-14

10. Mercola M, Ruiz-Lozano P, Schneider MD (2011) Cardiac muscle regeneration: lessons from development. Genes Dev 25, 299-309

11. Nascone $N$ and Mercola $M$ (1995) An inductive role for the endoderm in Xenopus cardiogenesis. Development $121,515-523$

12. Kim C, Majdi M, Xia P et al (2010) Non-cardiomyocytes influence the electrophysiological maturation of human embryonic stem cell-derived cardiomyocytes during differentiation. Stem Cells Dev 19, 783-795

13. Filipczyk AA, Passier R, Rochat A et al (2007) Regulation of cardiomyocyte differentiation of embryonic stem cells by extracellular signalling. Cell Mol Life Sci 64, 704-718

14. Yang L, Soonpaa MH, Adler ED et al (2008) Human cardiovascular progenitor cells develop from a KDR + embryonic-stem-cell-derived population. Nature 453, 524-528

15. Lian X, Hsiao C, Wilson G et al (2012) Robust cardiomyocyte differentiation from human pluripotent stem cells via temporal modulation of canonical Wnt signaling. Proc Natl Acad Sci U S A 109, E1848-1857

16. Moretti A, Bellin M, Welling A et al (2010) Patient-specific induced pluripotent stem-cell models for long-QT syndrome. N Engl J Med 363, 1397-1409

17. Itzhaki I, Maizels L, Huber I et al (2011) Modelling the long QT syndrome with induced pluripotent stem cells. Nature 471, 225-229

18. Yazawa M, Hsueh B, Jia X et al (2011) Using induced pluripotent stem cells to investigate cardiac phenotypes in Timothy syndrome. Nature 471, 230-234

19. Ruan Y, Liu N, Napolitano C and Priori SG (2008) Therapeutic strategies for long-QT syndrome: does the molecular substrate matter? Circ Arrhythm Electrophysiol 1, 290-297

20. Terrenoire C, Wang K, Tung KW et al (2013) Induced pluripotent stem cells used to reveal drug actions in a long 
QT syndrome family with complex genetics. J Gen Physiol 141, 61-72

21. Moreno JD and Clancy CE (2012) Pathophysiology of the cardiac late $\mathrm{Na}$ current and its potential as a drug target. J Mol Cell Cardiol 52, 608-619

22. Carvajal-Vergara X, Sevilla A, D'Souza SL et al (2010) Patient-specific induced pluripotent stem-cell-derived models of LEOPARD syndrome. Nature 465, 808-812

23. Lan F, Lee AS, Liang P et al (2013) Abnormal calcium handling properties underlie familial hypertrophic cardiomyopathy pathology in patient-specific induced pluripotent stem cells. Cell Stem Cell 12, 101-113

24. Tabar V and Studer L (2014) Pluripotent stem cells in regenerative medicine: challenges and recent progress. Nat Rev Genet 15, 82-92

25. Zhang SC, Wernig M, Duncan ID, Brüstle $O$ and Thomson JA (2001) In vitro differentiation of transplantable neural precursors from human embryonic stem cells. Nat Biotechnol 19, 1129-1133

26. Reubinoff $B E$, Itsykson $P$, Turetsky $T$ et al (2001) Neural progenitors from human embryonic stem cells. Nat Biotechnol 19, 1134-1140

27. Chambers SM, Fasano CA, Papapetrou EP, Tomishima M, Sadelain M and Studer L. (2009) Highly efficient neural conversion of human ES and iPS cells by dual inhibition of SMAD signaling. Nat Biotechnol 27, 275-280

28. Kondo T, Asai M, Tsukita K et al (2013) Modeling Alzheimer's disease with iPSCs reveals stress phenotypes associated with intracellular Abeta and differential drug responsiveness. Cell Stem Cell 12, 487-496

29. Yagi T, Ito D, Okada Y et al (2011) Modeling familial Alzheimer's disease with induced pluripotent stem cells. Hum Mol Genet 20, 4530-4539

30. Wang H and Doering LC (2012) Induced pluripotent stem cells to model and treat neurogenetic disorders. Neural Plast 2012, 346053

31. Israel MA, Yuan SH, Bardy $C$ et al (2012) Probing sporadic and familial Alzheimer's disease using induced pluripotent stem cells. Nature 482, 216-220

32. Brennand KJ, Simone A, Jou J et al (2011) Modelling schizophrenia using human induced pluripotent stem cells. Nature 473, 221-225

33. Ryan SD, Dolatabadi N, Chan SF et al (2013) Isogenic human iPSC Parkinson's model shows nitrosative stress-induced dysfunction in MEF2-PGC1alpha transcription. Cell 155, 1351-1364

34. Kaye JA and Finkbeiner S (2013) Modeling Huntington's disease with induced pluripotent stem cells. Mol Cell Neurosci 56, 50-64

35. Jeon I, Lee N, Li JY et al (2012) Neuronal properties, in vivo effects, and pathology of a Huntington's disease patient-derived induced pluripotent stem cells. Stem Cells 30, 2054-2062

36. Zhang K, Yi F, Liu GH and Izpisua Belmonte JC (2012) Huntington's disease: dancing in a dish. Cell Res 22, 1627-1630

37. Williams EC, Zhong X, Mohamed A et al (2014) Mutant astrocytes differentiated from Rett syndrome patients-specific iPSCs have adverse effects on wild-type neurons. Hum Mol Genet 23, 2968-2980
38. Jeon I, Choi C, Lee $\mathrm{N}$ et al (2014) In Vivo Roles of a Patient-Derived Induced Pluripotent Stem Cell Line (HD72-iPSC) in the YAC128 Model of Huntington's Disease. Int J Stem Cells 7, 43-47

39. Yang J, Cai J, Zhang Y et al (2010) Induced pluripotent stem cells can be used to model the genomic imprinting disorder Prader-Willi syndrome. J Biol Chem 285, 4030340311

40. Dajani R, Koo SE, Sullivan GJ and Park IH (2013) Investigation of Rett syndrome using pluripotent stem cells. J Cell Biochem 114, 2446-2453

41. Ananiev G, Williams EC, Li H and Chang Q (2011) Isogenic pairs of wild type and mutant induced pluripotent stem cell (iPSC) lines from Rett syndrome patients as in vitro disease model. PLoS One 6, e25255

42. Schöndorf DC, Aureli M, McAllister FE et al (2014) iPSC-derived neurons from GBA1-associated Parkinson's disease patients show autophagic defects and impaired calcium homeostasis. Nat Commun 5, 4028

43. Chamberlain SJ, Chen PF, Ng KY et al (2010) Induced pluripotent stem cell models of the genomic imprinting disorders Angelman and Prader-Willi syndromes. Proc Natl Acad Sci U S A 107, 17668-17673

44. Sareen D, Ebert AD, Heins BM, McGivern JV, Ornelas L and Svendsen CN (2012) Inhibition of apoptosis blocks human motor neuron cell death in a stem cell model of spinal muscular atrophy. PLoS One 7, e39113

45. Egawa $\mathrm{N}$ and Inoue $\mathrm{H}$ (2013) [ALS disease modeling and drug screening using patient-specific iPS cells]. Rinsho Shinkeigaku 53, 1020-1022

46. Corti S, Nizzardo M, Simone C et al (2012) Genetic correction of human induced pluripotent stem cells from patients with spinal muscular atrophy. Sci Transl Med 4, 165ra162

47. Ross CA and Akimov SS (2014) Human-induced pluripotent stem cells: potential for neurodegenerative diseases. Hum Mol Genet 23, R17-26

48. Kiskinis E, Sandoe J, Williams LA et al (2014) Pathways Disrupted in Human ALS Motor Neurons Identified through Genetic Correction of Mutant SOD1. Cell Stem Cell 14, 781-795

49. Chen H, Qian K, Du Z et al (2014) Modeling ALS with iPSCs Reveals that Mutant SOD1 Misregulates Neurofilament Balance in Motor Neurons. Cell Stem Cell 14, 796-809

50. Wolstencroft EC, Mattis V, Bajer AA, Young PJ and Lorson $\mathrm{CL}$ (2005) A non-sequence-specific requirement for SMN protein activity: the role of aminoglycosides in inducing elevated SMN protein levels. Hum Mol Genet 14, 1199-1210

51. Makhortova NR, Hayhurst M and Cerqueira A (2011) A screen for regulators of survival of motor neuron protein levels. Nat Chem Biol 7, 544-552

52. Guo X, Disatnik MH, Monbureau M, Shamloo M, Mochly -Rosen D and Qi X (2013) Inhibition of mitochondrial fragmentation diminishes Huntington's disease-associated neurodegeneration. J Clin Invest 123, 5371-5388

53. Charbord J, Poydenot P, Bonnefond C et al (2013) High throughput screening for inhibitors of REST in neural derivatives of human embryonic stem cells reveals a chem- 
ical compound that promotes expression of neuronal genes. Stem Cells 31, 1816-1828

54. Miller JD, Ganat YM, Kishinevsky S et al (2013) Human iPSC-based modeling of late-onset disease via progerin-induced aging. Cell Stem Cell 13, 691-705

55. Kim C, Wong J, Wen J et al (2013) Studying arrhythmogenic right ventricular dysplasia with patient-specific iPSCs. Nature 494, 105-110

56. Reinhardt P, Schmid B, Burbulla LF et al (2013) Genetic correction of a LRRK2 mutation in human iPSCs links parkinsonian neurodegeneration to ERK-dependent changes in gene expression. Cell Stem Cell 12, 354-367

57. Lopaschuk GD and Jaswal JS (2010) Energy metabolic phenotype of the cardiomyocyte during development, differentiation, and postnatal maturation. J Cardiovasc Pharmacol 56, 130-140

58. Djouadi F, Lecarpentier Y, Hébert JL, Charron P, Bastin J and Coirault C (2009) A potential link between peroxisome proliferator-activated receptor signalling and the pathogenesis of arrhythmogenic right ventricular cardiomyopathy. Cardiovasc Res 84, 83-90

59. Eroshenko N, Ramachandran R, Yadavalli VK and Rao RR (2013) Effect of substrate stiffness on early human embryonic stem cell differentiation. J Biol Eng 7, 7

60. Engler AJ, Carag-Krieger C, Johnson CP et al (2008) Embryonic cardiomyocytes beat best on a matrix with heartlike elasticity: scar-like rigidity inhibits beating. J Cell Sci 121, 3794-3802

61. Galie PA, Khalid N, Carnahan KE, Westfall MV and Stegemann JP (2013) Substrate stiffness affects sarcomere and costamere structure and electrophysiological function of isolated adult cardiomyocytes. Cardiovasc Pathol 22, 219-227

62. Choi SM, Kim Y, Shim JS et al (2013) Efficient drug screening and gene correction for treating liver disease using patient-specific stem cells. Hepatology 57, 24582468

63. Hidvegi T, Ewing $M$, Hale $P$ et al (2010) An autophagy-enhancing drug promotes degradation of mutant alpha1-antitrypsin $\mathrm{Z}$ and reduces hepatic fibrosis. Science 329, 229-232

64. Höing S, Rudhard Y, Reinhardt P et al (2012) Discovery of inhibitors of microglial neurotoxicity acting through multiple mechanisms using a stem-cell-based phenotypic assay. Cell Stem Cell 11, 620-632
65. Yang YM, Gupta SK, Kim KJ et al (2013) A small molecule screen in stem-cell-derived motor neurons identifies a kinase inhibitor as a candidate therapeutic for ALS. Cell Stem Cell 12, 713-726

66. Fermini B and Fossa AA (2003) The impact of drug-induced QT interval prolongation on drug discovery and development. Nat Rev Drug Discov 2, 439-447

67. Zeevi-Levin N, Itskovitz-Eldor J and Binah O (2012) Cardiomyocytes derived from human pluripotent stem cells for drug screening. Pharmacol Ther 134, 180-188

68. De Bruin ML, Pettersson M, Meyboom RH, Hoes AW and Leufkens HG (2005) Anti-HERG activity and the risk of drug-induced arrhythmias and sudden death. Eur Heart J 26, 590-597

69. Liang P, Lan F, Lee AS et al (2013) Drug screening using a library of human induced pluripotent stem cell-derived cardiomyocytes reveals disease-specific patterns of cardiotoxicity. Circulation 127, 1677-1691

70. Grant AO (2009) Cardiac ion channels. Circ Arrhythm Electrophysiol 2, 185-194

71. Knollmann BC (2013) Induced pluripotent stem cell-derived cardiomyocytes: boutique science or valuable arrhythmia model? Circ Res 112, 969-976; discussion 976

72. Musunuru K (2013) Genome editing of human pluripotent stem cells to generate human cellular disease models. Dis Model Mech 6, 896-904

73. Kim H and Kim JS (2014) A guide to genome engineering with programmable nucleases. Nat Rev Genet 15, 321-334

74. Maeder ML, Linder SJ, Cascio VM, Fu Y, Ho QH and Joung JK (2013) CRISPR RNA-guided activation of endogenous human genes. Nat Methods 10, 977-979

75. Qiang L, Fujita R and Abeliovich A (2013) Remodeling neurodegeneration: somatic cell reprogramming-based models of adult neurological disorders. Neuron 78, 957-969

76. Wada R, Muraoka N, Inagawa K et al (2013) Induction of human cardiomyocyte-like cells from fibroblasts by defined factors. Proc Natl Acad Sci U S A 110, 12667-12672

77. Fu JD, Stone NR, Liu L et al (2013) Direct Reprogramming of Human Fibroblasts toward a Cardiomyocyte-like State. Stem Cell Reports 1, 235-247

78. Cerrone M, Lin X, Zhang M et al (2014) Missense mutations in plakophilin-2 cause sodium current deficit and associate with a brugada syndrome phenotype. Circulation $129,1092-1103$ 